\title{
State-Dependent Implication and Equivalence in Quantum Logic
}

\section{Fedor Herbut}

Department of Mathematics, Physics, and Geological Sciences, Serbian Academy of Sciences and Arts, Knez Mihajlova 35, 11000 Belgrade, Serbia

Correspondence should be addressed to Fedor Herbut, fedorh@sanu.ac.rs

Received 29 March 2012; Accepted 9 September 2012

Academic Editor: Emilio Elizalde

Copyright (C) 2012 Fedor Herbut. This is an open access article distributed under the Creative Commons Attribution License, which permits unrestricted use, distribution, and reproduction in any medium, provided the original work is properly cited.

Ideal occurrence of an event (projector) $E$ leads to the known change of a state (density operator) $\rho$ into $E \rho E /[\operatorname{tr}(E \rho)]$ (the Lüders state). It is shown that two events $E$ and $F$ give the same Lüders state if and only if the equivalence relation $E \rho=F \rho$ is valid. This relation determines equivalence classes. The set of them and each class, are studied in detail. It is proved that the range projector $\bar{Q}$ of the Lüders state can be evaluated as $\bar{Q}=E-\left(E \wedge Q_{0}\right)$, where $\bigwedge$ denotes the greatest lower bound, and $Q_{0}$ is the null projector of $\rho$. State-dependent implication $\leq_{\rho}$ extends absolute implication (which, in turn, determines the entire structure of quantum logic). $\bar{Q}$ and $\leq_{\rho}$ are investigated in a closely related way to mutual benefit. Inherent in the preorder $\leq_{\rho}$ is the state-dependent equivalence $\sim \rho$, defining equivalence classes in a given Boolean subalgebra. The quotient set, in which the classes are the elements, has itself a partially ordered structure, and so has each class. In a complete Boolean subalgebra, both structures are complete lattices. Physical meanings are discussed.

\section{Introduction}

The basic object of this study is the concept of a quantum-mechanical state (density operator) $\rho$ and its change when an event (projector) $E$ with positive probability $\operatorname{tr}(\rho E)>0$ occurs (the result 1 is obtained) in ideal measurement. The terms "state" and "density operator" as well as "event," and "projector" will be used interchangeably.

As it is well known, ideal measurement is the simplest special case of measurement of the first kind (synonyms: predictive, repeatable, and nondemolition measurement). It causes a change of state according to the Lüders selective (or definite-result) formula

$$
\rho \longrightarrow \bar{\rho} \equiv \frac{E \rho E}{[\operatorname{tr}(\rho E)]}
$$


See [1-3]. We call $\bar{\rho}$ the Lüders state, and $E$ a state-determining projector (it is all meant with respect to the initially given state $\rho$ ).

Usually one treats the special case of a pure-state $\rho=|\psi\rangle\langle\psi|$. Then, as easily seen, (1.1) takes the simple form

$$
|\psi\rangle \longrightarrow \frac{E|\psi\rangle}{(\langle\psi|E| \psi\rangle)^{1 / 2}}
$$

which is sometimes called the von Neumann-Lüders projection (it is actually a normalized projection). Von Neumann treated the even more special case when the event is elementary (an atom) $E=|\phi\rangle\langle\phi|[4]$. The change of state then is

$$
|\psi\rangle \longrightarrow|\phi\rangle
$$

The Lüders state was postulated by Lüders. It was derived by several authors including the present one $[5,6]$ (and the first derivation was repeated in different context in [7]; see also references in these articles).

For a different approach, see references [8-10]. In Khrennikov's terminology, one deals with the postulates of Lüders and von Neumann (and he carefully examines their effect on some foundational issues).

As it was mentioned, in my view, ideal measurement is the simplest kind of measurement, and of which kind a measurement is depends on the interaction of object and measuring instrument (an elaboration of this will be presented in a followup). Both Lüders and von Neumann treat ideal measurement, but the former allows degenerate eigenvalues of the measured observable, whereas the latter is confined to the special case of complete observables, that is, to ones with all eigenvalues nondegenerate.

Incidentally, I have shown in my mentioned article [6] (see Section 3 there) that the Lüders change of state can be obtained by measuring a suitable complete observable as its refinement (in the way of von Neumann), but the choice of the latter depends on the state in which the measurement is performed. Khrennikov has rediscovered essentially the same result independently in [9].

Change of state (1.1) is made use of in the concept of state-dependent (SD) implication $E \leq_{\rho} F$, where $E$ and $F$ are events, and $\rho$ is a given state [11-13]. Absolute (or state independent) implication $E \leq F \Leftrightarrow E F=E$ in quantum logic $D(\mathscr{l})$ (the complete lattice of all projectors in the Hilbert space $\mathscr{H}$ of the given quantum system) is such that if an event $E$ occurs in a state $\rho$, that is, if $\operatorname{tr}(\rho E)=1$, then also the implied event $F$ occurs; and this is so for every state.

SD implication $\leq_{\rho}$, which is a generalization if the absolute implication (cf. Corollary 2.3 below), is, on the other hand, based on the following reasoning. If an event $E$ occurs in an ideal way in a given state $\rho$ and, after the occurrence, another event $F$ ipso facto also occurs, then one says that $E$ implies state-dependently $F$ and one writes $E \leq_{\rho} F$.

When one takes into account that ideal occurrence gives rise to (1.1), one obtains the equivalence relation

$$
E \leq_{\rho} F \Longleftrightarrow \operatorname{tr}\left[\left(\frac{E \rho E}{[\operatorname{tr}(\rho E)]}\right) F\right]=1
$$


Evidently, the SD implication relation (1.4) is restricted to nonzero-probability statedependently implying events $E$.

$\mathrm{SD}$ implication is defined on a given Boolean subalgebra $\mathcal{B}$ of $P(\mathscr{\ell})$ (to achieve transitivity) [11]. SD implication on $B$ is a preorder [11,14]. As every preorder, being reflexive and transitive but not antisymmetric, SD implication defines an equivalence relation on $B, S D$ equivalence $\sim \rho$, as follows:

$$
E \sim{ }_{\rho} F \Longleftrightarrow E \leq{ }_{\rho} F, \quad F \leq_{\rho} E
$$

$\mathrm{SD}$ equivalence breaks up $\mathbb{B}$ into equivalence classes, the elements of the $\mathrm{SD}$ quotient set $B / \sim \rho$. By this all zero-probability projectors $\{E: \operatorname{tr}(\rho E)=0\}$ are taken to form one class. (This will be seen to be correct, cf. Remark 2.2.) The SD equivalence class in $B$ to which a given projector $E$ belongs will be denoted by $[E]_{\mathcal{B}}$.

The SD implication concept is further elaborated in the next section. But, unlike in $[11,12]$, this time the powerful structure of Boolean algebras is not made use of. Only poset (partially ordered set) theory is utilized, and poset structure is introduced in the SD quotient set $B / \sim \rho$.

\section{SD Implication Further Elaborated}

Simple algebraic relations characterizing SD implication and SD equivalence are now derived.

Proposition 2.1. If $\operatorname{tr}(E \rho)>0$, then $E \leq_{\rho} F$ if and only if $E \rho=E F \rho$ is valid.

Proof. On account of commutation under the trace, idempotency of projectors, and commutation in $\boldsymbol{B}$, the mentioned former definition (1.4) of state-dependent implication boils down to $\operatorname{tr}(E \rho)=\operatorname{tr}(E F \rho)$, or equivalently to $\operatorname{tr}[(E-E F) \rho]=0$. Since $(E-E F)$ is a projector, one can further equivalently write $\operatorname{tr}[(E-E F) \rho(E-E F)]=0$, and $(E-E F) \rho(E-E F)=0$ (no other positive operator then zero gives trace zero). Finally the Lüders lemma (page 326 in [1], cf. also Remark 4.3) gives $(E-E F) \rho=0$.

Remark 2.2. Relation (1.4) as the definition of SD implication cannot be extended to projectors $E$ for which $E \rho=0$, but the equivalent definition $E \rho=E F \rho$ for $E \rho \neq 0$ can. Hence, we extend formally SD implication to these projectors. If an event $E$ is such that $\operatorname{tr}(\rho E)=0$, then it (formally) state-dependently implies every other event in $乃$. This statement cannot be falsified because $E$ cannot occur in $\rho$. Hence it is formally true though trivial (more on this in Lemma 4.7).

One should note the equality of sets $\{E: E \rho=0\}=\{E: \operatorname{tr}(\rho E)=0\}$ due to $0=\operatorname{tr}(\rho E)=$ $\operatorname{tr}(E \rho E) \Rightarrow 0=E \rho E=E \rho$ (cf. for analogy proof of Proposition 2.1).

Corollary 2.3. SD implication generalizes and extends absolute implication, that is, the latter is a special case of the former, and the former can be valid for pairs of events for which the latter is not valid.

Proof. If $E=E F$, then relation (1.4) is obviously valid, and $E \leq_{\rho} F$ ensues. 
SD implication $\leq_{\rho}$, being reflexive, transitive but not antisymmetric, is a partial order [14]. As for every partial order, one can take the SD (equivalence) quotient set $B / \sim \rho$, the elements of which are the SD equivalence classes $[E]_{\mathcal{B}}$ in $\mathcal{B}$. Next, one can induce the corresponding partial-order relation in the quotient set and thus obtain a proper binary relation for quantum logic. based.

We proceed by acquiring a simple insight on which the poset structure of $B / \sim_{\rho}$ is

Proposition 2.4. Let $[E]_{B}$ and $[F]_{B}$ be distinct $S D$ equivalence classes, that is, elements of $B / \sim_{\rho}$, such that $\exists E^{\prime} \in[E]_{\mathcal{B}}$ and $\exists F^{\prime} \in[F]_{\mathcal{B}}$ so that $E^{\prime} \leq_{\rho} F^{\prime}$. Let further $E^{\prime \prime}$ and $F^{\prime \prime}$ be arbitrary elements in the same respective classes. Then also $E^{\prime \prime} \leq_{\rho} F^{\prime \prime}$. In other words, if one projector from one class implies state dependently a projector from another class, then every projector from the former class implies state dependently every projector from the latter one.

Proof. The very definition of the classes implies $E^{\prime \prime} \leq_{\rho} E^{\prime}$ and $F^{\prime} \leq_{\rho} F^{\prime \prime}$. In view of the assumed relation $E^{\prime} \leq_{\rho} F^{\prime}$, and due to the validity of transitivity of SD implication in $B, E^{\prime \prime} \leq_{\rho} F^{\prime \prime}$ follows.

On ground of Proposition 2.4 we can define a poset structure in $B / \sim \rho$.

Definition 2.5. One has $[E]_{\mathcal{B}} \leq_{\rho}[F]_{\mathcal{B}}$ if for all $E^{\prime} \in[E]_{\mathcal{B}}$ and for all $F^{\prime} \in[F]_{\mathcal{B}}$ the relation $E^{\prime} \leq_{\rho} F^{\prime}$ is valid.

Lemma 2.6. SD implication thus defined in the $S D$ quotient set $B / \sim_{\rho}$ is a partial order relation, that is, it has the properties of reflexivity, transitivity, and antisymmetry.

Proof. Reflexivity. If $[E]_{\mathcal{B}}$ is an arbitrary SD equivalence class in $\mathcal{B}$, and $E$ is its arbitrary element, one has $E \leq_{\rho} E$ and $E \geq_{\rho} E$, so that, according to Definition 2.5, also $[E]_{B} \leq_{\rho}[E]_{B}$ and $[E]_{B} \geq_{\rho} E_{\bar{B}}$ are valid.

Transitivity. If $[E]_{B} \leq_{\rho}[F]_{\mathcal{B}}$ and $[F]_{B} \leq_{\rho}[G]_{B}$, and $E^{\prime}, F^{\prime}, G^{\prime}$ are arbitrary elements from the respective classes, then, according to Proposition 2.4, the relations $E^{\prime} \leq_{\rho} F^{\prime}$ and $F^{\prime} \leq_{\rho} G^{\prime}$ hold true. Transitivity of SD implication in $B$ then implies the same for the SD quotient set: from $E^{\prime} \leq_{\rho} G^{\prime}$ ensues $[E]_{\mathcal{B}} \leq_{\rho}[G]_{\mathcal{B}}$.

Antisymmetry. Let $[E]_{\mathcal{B}} \leq_{\rho}[F]_{\mathcal{B}}$ and $[F]_{B} \leq_{\rho}[E]_{\mathcal{B}}$ be valid. Then Definition 2.5 implies $E \leq_{\rho} F$ and $F \leq_{\rho} E$, having $E \sim_{\rho} F$ as its consequence, that is, one obtains $[E]_{B}=[F]_{B}$.

Remark 2.7. Having in mind that $\mathbb{B}$ can be arbitrarily chosen in $D(\mathscr{\ell})$, we may call the SD quotient set $B / \sim_{\rho}$ SD quantum logic paralleling the set of all projectors, quantum logic $p(\mathscr{l})$. Since the term "quantum logic" has also another meaning as a branch of mathematical physics, one might use the term "SD quantum logic" also to designate a part of quantum logic (meant as the mentioned branch), the part treated in [11-13], and in this paper (cf. also the last passage in Section 11).

Proposition 2.8. Two projectors $E, F \in B$ are state-dependently equivalent $E \sim \rho F$ if and only if $E \rho=$ $F \rho$ is satisfied.

Proof. Relation (1.5) and Proposition 2.1 imply $E \rho=E F \rho$ and $F \rho=F E \rho$. Since all projectors in $B$ commute, necessity of the claimed condition obviously follows. As to sufficiency, the claimed condition and idempotency obviously imply $E \rho=E F \rho$ and $F \rho=F E \rho$, which, in view of Proposition 2.1 and relation (1.5), is tantamount to $E \sim{ }_{\rho} F$. 
Remark 2.9. The SD quotient set $\mathbb{B} / \sim \rho$ is complemented because orthocomplementation in $\mathbb{B}$ takes each SD equivalence class onto another, and none is self-complemented. Finite Boolean subalgebras thus consist of an even number of elements.

Evidently, SD equivalence $E \sim \rho F(\Leftrightarrow E \rho=F \rho)$ can be extended to the entire quantum logic $P(\mathscr{\ell})$. In what immediately follows we study SD equivalence in the entire $P(\mathscr{\ell})$, and we write the equivalence classes as $[E]$ (without index). Subsequently, beginning with Section 8 , we shall return to restricting our investigation to $B$ to obtain additional insight in SD quantum logic.

\section{Lüders States}

We now express SD implication in terms of a (known but, perhaps, not well known) projector relation.

Lemma 3.1. Denoting by $Q$ the range projector of a given density operator $\rho$, the general claim is valid that an event $E$ has probability 1 in the state $\rho$ if and only if $Q \leq E$.

Proof. The proof is given for the reader's convenience in Appendix A.

Corollary 3.2. Denoting the range projector of the Lüders state $\bar{\rho}$ (cf. (1.1)) by $\bar{Q}$, and calling it Lüders projector, relation (1.4) and Lemma 3.1 entail the following necessary and sufficient condition for SD implication in terms of it:

$$
E \leq_{\rho} F \Longleftrightarrow \bar{Q} \leq F
$$

Evidently, the projector $E$ in Corollary 3.2 is somehow inherent in $\bar{\rho}$. We want to find out how, and proceed by evaluating the Lüders projector $\bar{Q}$. One needs, not $Q$, the initial range projector (of the initially given state $\rho$ ), but its ortho-complementary projector, the null-space projector, which one denotes by $Q_{0}$ and call the initial null projector. Besides, one shall need more insight into the properties of $E$ that appears in (1.1). As stated, one calls such a projector a state determining one.

Lemma 3.3. Let $E$ and $\rho$ be an arbitrary projector and an arbitrary density operator, respectively. Then the following equivalence relation is valid

$$
\operatorname{tr}(E \rho)=1 \Longleftrightarrow E \rho=\rho
$$

This general equivalence is known, but perhaps not well known. For the reader's convenience it is proved in Appendix B.

Corollary 3.4. Let $E$ be a state determining projector. Then, obviously $E \bar{\rho}=\bar{\rho}(c f .(1.1)$ ), and, on account of Lemmas 3.3 and 3.1, one has

$$
\bar{Q} \leq E
$$


Definition 3.5. On account of Corollary 3.4, we can define a new projector $\bar{E}$ as follows:

$$
\bar{E} \equiv E-\bar{Q} .
$$

We call $\bar{E}$ the redundancy projector (for reasons that will become clear below).

Proposition 3.6. The redundancy projector $\bar{E}$ of a state-determining projector $E$ in (1.1) satisfies both $\bar{E} \leq \bar{Q}_{0}$ and $\bar{E} \leq Q_{0}$, where $\bar{Q}_{0} \equiv I-\bar{Q}$, I being the identity operator, is the Lüders null projector and $Q_{0}$ is the initial null projector (that of $\rho$ ).

Proof. Definition 3.5 makes $\bar{E}$ orthogonal to $\bar{Q}$, hence $\bar{E} \leq \bar{Q}_{0}$ it must be a subprojector of the null projector $\bar{Q}_{0}, \bar{E} \leq \bar{Q}_{0}$, because $\bar{Q}_{0}=I-\bar{Q}$, where $I$ is the identity operator.

Let $|\phi\rangle \in \mathcal{R}\left(\bar{Q}_{0}\right)$, where $\mathcal{R}\left(\bar{Q}_{0}\right)$ is the range of $\bar{Q}_{0}$, that is, the null space of the Lüders state (cf. (1.1)). Further, let $\rho=\sum_{i} r_{i}|i\rangle\langle i|$ be a spectral decomposition of the initially given density operator $\rho$ into eigen-ray-projectors with all eigenvalues $\left\{r_{i}: \forall i\right\}$ positive. This is always possible. Then, in view of (1.1),

$$
0=\langle\phi|E \rho E| \phi\rangle=\sum_{i} r_{i}\langle\phi|E| i\rangle\langle i|E| \phi\rangle=\sum_{i} r_{i}|\langle i|E| \phi\rangle|^{2},
$$

implying

$$
\forall i:\langle i|E| \phi\rangle=0 \text {. }
$$

Since $|\phi\rangle$ is any state vector in $\mathcal{R}\left(\bar{Q}_{0}\right)$, and the vectors $\{|i\rangle: \forall i\}$ span the range of the initial state $\rho$, the last relations mean that the image subspace $E\left[\mathcal{R}\left(\bar{Q}_{0}\right)\right]$ is orthogonal to $\mathcal{R}(\rho)$, that is, that $E$ maps the null space $\mathcal{R}\left(\bar{Q}_{0}\right)$ into the null space $\mathcal{R}\left(Q_{0}\right)$ :

$$
E\left[\mathcal{R}\left(\bar{Q}_{0}\right)\right] \subseteq \mathcal{R}\left(Q_{0}\right)
$$

Further, $\bar{E}$ being a subprojector of $\bar{Q}_{0}$, one has $\mathcal{R}(\bar{E}) \subseteq \mathcal{R}\left(\bar{Q}_{0}\right)$. Finally, since $\bar{E}$ is also a subprojector of the idempotent operator $E$ (cf. Definition 3.5), it follows that

$$
E[\mathcal{R}(\bar{E})]=\mathcal{R}(\bar{E}) \subseteq E\left[\mathcal{R}\left(\bar{Q}_{0}\right)\right]
$$

Therefore, transitivity of the subset relation implies $\mathcal{R}(\bar{E}) \subseteq \mathcal{R}\left(Q_{0}\right)$, or equivalently $\bar{E} \leq Q_{0}$ as claimed.

Theorem 3.7. The Lüders projector $\bar{Q}$ can be evaluated in terms of a state determining projector $E$ (cf. (1.1)) and the initial null projector $Q_{0}$ as follows:

$$
\bar{Q}=E-\left(E \wedge Q_{0}\right),
$$

where $E \wedge Q_{0}$ is the greatest lower bound (glb) or largest common subprojector of $E$ and $Q_{0}$. 
Proof. Let us introduce $G \equiv E-\left(E \wedge Q_{0}\right)$ or equivalently

$$
E=\left(E \wedge Q_{0}\right)+G
$$

Since $\left(E \wedge Q_{0}\right)$ is a sub-projector of the initial null projector $Q_{0}$, one has $\left(E \wedge Q_{0}\right) \rho=0=\rho\left(E \wedge Q_{0}\right)$ implying

$$
G \rho G=E \rho E .
$$

Hence, also $G$ is a determining projector for $\bar{\rho}$ (cf. (1.1)), and all that has been proved for the determining projector $E$ in (1.1) is valid also for $G$. In particular, $\bar{Q} \leq G, G \equiv \bar{Q}+\bar{G}$ (definition of $\bar{G}$ ).

The definition of $\bar{G}$ implies $\bar{G} \leq G$. On the other hand, it is seen from (3.10) that $G \leq E$. Hence, due to transitivity of absolute implication, $\bar{G} \leq E$. Since also Proposition 3.6 is valid for $G$ and its redundancy projector $\bar{G}$, one has $\bar{G} \leq Q_{0}$, and

$$
\bar{G} \leq\left(E \wedge Q_{0}\right)
$$

follows.

On can see from (3.10) that $G$ is orthogonal to $\left(E \wedge Q_{0}\right): G\left(E \wedge Q_{0}\right)=0$, and so is its subprojector $\bar{G}: \bar{G}\left(E \wedge Q_{0}\right)=(\bar{G} G)\left(E \wedge Q_{0}\right)=0$. The projector orthogonality amounts to $\bar{G} \leq$ $\left(E \wedge Q_{0}\right)^{c}$, where the suffix $c$ denotes taking the ortho-complementary projector. Comparing this with (3.12), we conclude that $\bar{G}=0$ because only zero is the common subprojector of a projector and its ortho-complementary projector.

Returning to the definition of $\bar{G}(\equiv G-\bar{Q})$, we see that we are led to the equality $G=\bar{Q}$, which, due to the definition (3.10) of $G$, completes the proof.

Theorem 3.7 immediately implies the following conclusions.

Corollary 3.8. The redundancy projector $\bar{E}$ of any state-determining projector $E \in P(\mathcal{H})$ is given by $\bar{E}=E \wedge Q_{0}$.

Corollary 3.9. A projector $E$ is a Lüders projector $\bar{Q}$ if and only if its redundancy projector is zero, and if and only if the only common subprojector of $E$ and $Q_{0}$ is zero:

$$
E=\bar{Q} \Longleftrightarrow \bar{E}=0 \Longleftrightarrow E \wedge Q_{0}=0
$$

Corollary 3.10. If $[E, \rho]=0$, or at least $\left[E, Q_{0}\right]=0$, then $\bar{Q}=E-E Q_{0}$.

Proof. The first commutation is equivalent, as well known, to reduction of $E$ in each eigensubspace of $\rho$. Since the null-space of $\rho$ is its eigen-subspace corresponding to the eigenvalue zero, $E$ reduces in it, hence it commutes with $Q_{0}$ that projects onto the latter. Then the glb equals the product as claimed.

Remark 3.11. Whenever $F \rho=E \rho$ is valid, one can replace $E$ by $F$ in the evaluation formula of the Lüders projector (cf. (1.1) and Theorem 3.7), that is, also $\bar{Q}=F-\left(F \wedge Q_{0}\right)$ is satisfied. 
Remark 3.12. If the initial density operator $\rho$ is nonsingular, that is, when $Q_{0}=0$, then the Lüders projector $\bar{Q}$ is the only state determining projector as obvious from Theorem 3.7.

\section{Generalized Lüders Lemma}

The SD equivalence relation $E \rho=F \rho$ implies that both $E$ and $F$ are state determining projectors for the same Lüders state $\bar{\rho}$ (cf. (1.1)). We want to clarify if the converse implication is also valid.

Theorem 4.1. Generalized Lüders lemma. If $E, F$ are two projectors and $\rho$ is a density operator, then $E \rho E=F \rho F$ implies $E \rho=F \rho$.

Proof. One can write $E=\bar{Q}+\bar{E}$ and $F=\bar{Q}+\bar{F}$ (cf. Definition 3.5). Further, on account of Proposition 3.6, both $\bar{E}$ and $\bar{F}$ are subprojectors of $Q_{0}$. Hence, $E \rho=(\bar{Q}+\bar{E}) \rho=\bar{Q} \rho$. On the other hand, symmetrically $F \rho=(\bar{Q}+\bar{F}) \rho=\bar{Q} \rho$.

Corollary 4.2. If $E \rho E=0$, then $E \rho=0$.

Proof. One can write $E \rho E=0 \rho 0$. Then Theorem 4.1 implies the claim.

Remark 4.3. The claim of Corollary 4.2 was proved by Lüders [1] (page 326) in the following more general form: If $B$ is a positive operator, and $C^{\dagger} B C=0$, where the dagger denotes adjoining (of the linear operator $C$ ), then $B C=0$ is valid. This auxiliary result is usually called "the Lüders lemma."

Lemma 4.4. The equivalence relation $E \sim \rho F$ gives, in general, finer equivalence classes in $D(\not d)$ then the equivalence relation $E \sim \operatorname{tr} \rho F \equiv(\operatorname{tr}(E \rho)=\operatorname{tr}(F \rho))$.

Proof. The proof is given by a simple example. We take the two-dimensional spin- $1 / 2$ space and we define: $\rho \equiv 1 / 2=(1 / 2)|+\rangle\langle+|+(1 / 2)|-\rangle\langle-|, E \equiv|+\rangle\langle+|, F \equiv|-\rangle\langle-|$. Then $E \rho=$ $(1 / 2)|+\rangle\langle+|\neq F \rho=(1 / 2)|-\rangle\langle-|$, though $\operatorname{tr}(E \rho)=1 / 2=\operatorname{tr}(F \rho)$. Thus, $E \sim \operatorname{tr} \rho F$ though $E \sim \rho F$ is not valid. The latter, finer, equivalence relation breaks up the coarser class $[E, F]_{\operatorname{tr} \rho}$, so that the two distinct finer classes $[E]_{B}$ and $[F]_{B}$ are within it.

Remark 4.5. Theorem 4.1 can be put as the claim that $E \rho E=F \rho F$ and $E \rho=F \rho$ are equivalent, that is, they both define the same equivalence relation $\sim_{\rho}$ in $D(\mathscr{\ell})$.

Lemma 4.6. Relation $E \rho=F \rho$ is valid if and only if $E$ and $F$ act equally on each element of the range of $\rho$. In particular, $S D$ equivalence is equivalent to $E Q=F Q$.

Proof. The general element of the range is of the form $\rho|\psi\rangle$, and SD equivalence in Proposition 2.8 amounts to $E \rho|\psi\rangle=F \rho|\psi\rangle$. This bears out the first claim of the lemma. Since the last relation is obviously equivalent to $E Q|\psi\rangle=F Q|\psi\rangle$, also the second claim is shown to hold true.

Lemma 4.7. One has $E \rho=0$, or equivalently $E \sim \rho 0$, if and only if $E Q_{0}=E$. The last relation means that $E$ is a subprojector of the initial null projector $Q_{0}$, that is, that $E \leq Q_{0}$ is valid. Thus, the $S D$ equivalence class $[0]=\left[Q_{0}\right]$ consists of all subprojectors of $Q_{0}$. The projectors 0 and $Q_{0}$ are the minimal and the maximal elements in the class, respectively, (cf. Remark 2.2). 
Proof. Sufficiency. Let the subprojector condition $E Q_{0}=E$ be valid. Then $E \rho=\left(E Q_{0}\right)(Q \rho)=0$. Necessity. Let $E \rho=0=0 \rho$ hold true. Then, according to Lemma 4.6, also $E Q=0 Q=0$ is true. Finally, $E=E Q+E Q_{0}=E Q_{0}$ as claimed.

Lemma 4.8. One has $E \rho=\rho$, or equivalently $E \sim \rho I$ if and only if $E Q=Q$. The last relation means that $Q$ is a subprojector of $E$, that is, that $Q \leq E$ is valid. Thus, the class $[Q]=[I]$ consists of all projectors of which $Q$ is a subprojector. The projectors $Q$ and the identity operator I are the minimal and the maximal elements of the class, respectively.

Proof. Follows immediately from Lemma 4.6.

Lemma 4.9. The equivalence $E \sim \rho$ implies $E \sim \rho G \sim \sim_{\rho} F$, where $G$ is defined as the common glb of $E$ and $F$.

Proof. It is known that $G \equiv E \wedge F=\lim _{n \rightarrow \infty}(E F E)^{n}$ (cf. solution 96 in [15]). On account of Proposition 2.8, the assumed equivalence relation (with the idempotencies of the projectors) implies $(E F E) \rho=E \rho$. Further, $(E F E)^{n} \rho=E \rho$ ensues. Finally, $G \rho=\left[\lim _{n \rightarrow \infty}(E F E)^{n}\right] \rho=$ $\lim _{n \rightarrow \infty} E \rho=E \rho$.

Proposition 4.10. Let $E \sim \rho$ F, and let by definition $H \equiv E-G, J \equiv F-G$ (cf. Lemma 4.9). Then $E \sim \sim_{\rho} F$ is equivalent to the simultaneous validity of the following two sub-projector relations

$$
H \leq Q_{0}, \quad J \leq Q_{0}
$$

Proof. Sufficiency. Let the two subprojector relations be valid. Then one can write $E=G+H Q_{0}$ and $F=G+J Q_{0}$. SD equivalence then immediately follows.

Necessity. $E \sim \rho F$ and Lemma 4.9 imply $E \rho=G \rho=F \rho$, and one has

$$
H \rho=(E-G) \rho=0=(F-G) \rho=J \rho .
$$

Proposition 4.10 has introduced the entities $H$ and $J$ in the general case. Incidentally, there is a general lemma that says: Two arbitrary projectors $E$ and $F$ commute if and only if $H$ and $J$ are orthogonal (since we actually do not need this claim, let its proof be left to the reader).

\section{Complete-Lattice Structure of the SD Equivalence Classes in $D(\mathscr{L})$}

We assume that an arbitrary density operator $\rho$ is given and subject to scrutiny the SD equivalence classes. On account of Remark 4.5 we know that each such class contains precisely all projectors that are state determining for one and the same Lüders state $\bar{\rho}$. We begin by paying attention to the Lüders projectors in the classes.

Proposition 5.1. (A) Each SD equivalence class $[E]$ contains a Lïders projector $\bar{Q}$, and it can be evaluated from any element $E$ of the class according to Theorem 3.7.

(B) Each Lüders projector $\bar{Q}$ is the unique minimal element in its class $[\bar{Q}]: E \sim \rho \bar{Q}$ implies $\bar{Q} \leq E$. 
(C) The following bijection $\widehat{b}$ maps the $S D$ quotient set $D(\mathscr{L}) / \sim_{\rho}$ onto the set of all Lüders projectors in $D(\mathscr{\ell})$, which we denote by $\mathcal{L}$ :

$$
\widehat{b}([E])=\bar{Q} \equiv E-\left(E \wedge Q_{0}\right), \quad[E] \in \frac{P(\mathscr{H})}{\sim_{\rho}}, \quad \widehat{b}^{-1}(\bar{Q})=[\bar{Q}], \quad \bar{Q} \in \mathcal{L} .
$$

Proof. (A) We assume that $[E]$ is any state-dependent equivalence class, and that $E$ is any of its elements. We take $\bar{Q}$ as defined by the relation in Theorem 3.7. Since $\left(E \wedge Q_{0}\right)$ is a subprojector of $Q_{0}$, it follows that we have $\left(E \wedge Q_{0}\right) \rho=0$, and then, according to the relation in Theorem 3.7, $\bar{Q} \rho=E \rho$. Thus, each class does contain at least one Lüders projector.

(B) That $\bar{Q} \leq E$ is immediately seen from Corollary 3.4. This makes $\bar{Q}$ the unique minimal element in $[E]$ (there cannot be two distinct minimal elements, because they would have to be subprojectors of each other, and $\leq$ is a partial order, the anti-symmetric property of which implies the equality).

(C) is now obvious.

It is now useful to be reminded of a general property of the complete lattice $P(\mathscr{\ell})$.

Lemma 5.2. Let $E$ be any nonzero projector. The set of all lower bounds $\left\{E^{\prime}: E^{\prime} \leq E\right\}$ is a complete lattice.

Though this a known and simple property of complete lattices, we prove it for the reader's convenience in Appendix C.

Theorem 5.3. An arbitrary SD equivalence class $[E]$, as a poset subset of $D(\mathscr{\ell})$, is isomorphic to the complete lattice $\left\{E^{\prime}: E^{\prime} \leq\left(Q_{0} \wedge \bar{Q}_{0}\right)\right\}$. The isomorphism $\hat{i}$ acts as follows:

$$
\widehat{i}(E) \equiv \bar{E}=E-\bar{Q}, \quad E \in[E] ; \quad \widehat{i}^{-1}\left(E^{\prime}\right)=\bar{Q}+E^{\prime}, \quad E^{\prime} \leq\left(Q_{0} \wedge \bar{Q}_{0}\right)
$$

Proof. Let $E^{\prime}, F^{\prime} \leq\left(\overline{Q^{c}} \wedge Q_{0}\right)$. Then, on account of the assumed orthogonalities $\bar{Q} E^{\prime}=0=\bar{Q} F^{\prime}$, one has

$$
\left(\bar{Q}+E^{\prime}\right)\left(\bar{Q}+F^{\prime}\right)=\bar{Q}+E^{\prime} F^{\prime}
$$

Thus, one has $\left(\bar{Q}+E^{\prime}\right)\left(\bar{Q}+F^{\prime}\right)=\left(\bar{Q}+E^{\prime}\right)$, symbolically $\left(\bar{Q}+E^{\prime}\right) \leq\left(\bar{Q}+F^{\prime}\right)$ if and only if $E^{\prime} F^{\prime}=E^{\prime}$, symbolically $E^{\prime} \leq F^{\prime}$.

We have the immediate consequence.

Corollary 5.4. Each $S D$ equivalence class is a complete lattice with the Lüders projector $\bar{Q}$ as the minimal element and $\bar{Q}+\left(Q_{0} \wedge \bar{Q}_{0}\right)$ as the maximal element.

The set of all Lüders projectors $\mathcal{L}$, as any subset of $D(\mathscr{\ell})$, is a poset. It has a lattice structure and it is easily connected with the ortho-complemented SD quotient set. 
Theorem 5.5. (A) The poset $\mathcal{L}$ has the structure of a complete glb-semilattice, the operation of glb (greatest lower bound) being the same as in the entire quantum logic $D(\mathcal{L})$. Its minimal element is the zero projector.

(B) There is a simple bijection $\widehat{\hat{i}^{\prime}}$ mapping $\mathcal{L}$ onto the $S D$ quotient set $D(\mathscr{H}) / \sim \rho$ that associates with each Lüders projector its SD equivalence class, and the inverse of which singles out the minimal element in the SD equivalence class:

$$
\widehat{i^{\prime}}(\bar{Q}) \equiv[\bar{Q}], \quad\left(\widehat{i^{\prime}}\right)^{-1}([E])=\bar{Q}, \quad \bar{Q} \sim{ }_{\rho} E
$$

The term "glb-semilattice" is consistent with the terminology of Birkhoff, who used the synonymous term "meet-semilattice" (cf. the definition on page 22 in [14]).

Proof of Theorem 5.5. Let $\left\{E_{m}: m \in M\right\}$ be any subset of $\mathcal{L}$, and let $E_{\mathrm{glb}} \equiv \wedge_{m \in M} E_{m}$ be the greatest lower bound of the projectors in the subset taken in $P(\mathscr{\ell})$. We show now that it is a Lüders projector. Corollary 3.9 implies that a projector is a Lüders projector if and only if only the zero projector is a common lower bound of it and $Q_{0}$.

We take $P$ as a common sub-projector of $E_{\mathrm{glb}}$ and of $Q_{0}$. On account of $E_{\mathrm{glb}}$ being a lower bound (a sub-projector) of each $E_{m}$ in the subset, $P$ is a common sub-projector of each $E_{m}$ and of $Q_{0}$. Since each $E_{m}$ is a Lüders projector, $P$ must be zero.

\section{Physical Meaning of Complete-Lattice Structure}

The physical meaning of an event (projector) $E$ consists in the fact that it can occur in a given state (density operator) $\rho$, when $\operatorname{tr}(E \rho)=1$, or "not occur" when $\operatorname{tr}(E \rho)=0$. In the latter case the opposite event (ortho-complementary event) $E^{c} \equiv I-E$ occurs. (There is a third possibility, $E$ can de indeterminate, that is, $0<\operatorname{tr}(E \rho)<1$. Then it is made to occur or not occur in an ensemble of measurements giving relative frequencies.)

Let $\left\{E_{m}: m \in M\right\}$ be an arbitrary set of projectors in $D(\mathscr{L})$. One may wonder if the set can, as a whole, occur? The answer is of course, that it can. This is the case when all the events in it occur. One may further wonder if this occurrence of the set of events can be equivalently replaced by occurrence of a single event. Affirmative answer is given by the following claim.

Proposition 6.1. The system of relations

$$
\forall m \in M: \operatorname{tr}\left(E_{m} \rho\right)=1
$$

is equivalent to the single relation $\operatorname{tr}\left[\left(\wedge_{m \in M} E_{m}\right) \rho\right]=1$.

Proof. Necessity. On account of Lemma 3.1, one can rewrite the system of relations in Proposition 6.1 equivalently as follows:

$$
\forall m \in M: Q \leq E_{m}
$$

Being a common lower bound of the set $\left\{E_{m}: m \in M\right\}, Q$ must satisfy $Q \leq \wedge_{m \in M} E_{m}$. This is equivalent, due to Lemma 3.1, $\operatorname{to} \operatorname{tr}\left[\left(\wedge_{m \in M} E_{m}\right) \rho\right]=1$. 
Sufficiency. Assuming $\operatorname{tr}\left[\left(\wedge_{m \in M} E_{m}\right) \rho\right]=1$, or equivalently $Q \leq\left(\wedge_{m \in M} E_{m}\right)$, and taking into account that $\forall m \in M:\left(\wedge_{m^{\prime} \in M} E_{m^{\prime}}\right) \leq E_{m}$, transitivity implies $\forall m \in M: Q \leq E_{m}$. This is equivalent to the system of relations in Proposition 6.1.

Let us turn to the other extreme case. Nonoccurrence of the set of events $\left\{E_{m}: m \in M\right\}$ also amounts to that of a single event.

Proposition 6.2. Non-occurrence of a set of events $\left\{E_{m}: m \in M\right\}$ is equivalent to non-occurrence of the single event $\vee_{m \in M} E_{m}$, where $\vee$ denotes the least upper bound.

Proof. The non-occurrence in question is equivalent to the occurrence of the set $\left\{E_{m}^{c}: m \in M\right\}$ of opposite events. This, in turn, is, according to Proposition 6.1, equivalent to the occurrence of $\wedge_{m \in M} E_{m}^{c}$. On account of the corresponding de Morgan rule, this projector can be rewritten as $\left(\vee_{m \in M} E_{m}\right)^{c}$. Occurrence of this event is equivalent to non-occurrence of the opposite event $\left(\vee_{m \in M} E_{m}\right)$.

Propositions 6.1 and 6.2 have made it clear that the physical meaning of the greatest lower bound and the least upper bound in a complete lattice is occurrence and nonoccurrence of a set of events, respectively.

\section{Physical Meaning of the Complete-Lattice Structure of the SD Equivalence Classes}

We have seen that every SD equivalence class contains precisely all those events, that, as projectors, are the state-determining ones for one and the same Lüders state. These events have the same probability in the initially given state $\rho$, and, more importantly, they give rise to the same change of state in ideal measurement. In this sense, they are indistinguishable, and the SD equivalence class that they constitute, as a whole, correctly replaces its individual elements.

According to Proposition 6.1, the minimal element in the class, which is its glb, and which is the Lüders projector $\bar{Q}$, determines via its occurrence the occurrence of the whole class. Its maximal element $\bar{Q}+\left(Q_{0} \wedge \bar{Q}_{0}\right)$, via its non-occurrence determines that of the entire class. Thus, the two extreme elements in the class make possible the occurrence or nonoccurrence of the class as a set of indistinguishable events (as far as probability and ideal measurement go).

One wonders if the elements of the class that are intermediate between the extreme ones do have any physical meaning. They are distinguished via the redundancy projectors

$$
\bar{E} \in\left\{E^{\prime}: E^{\prime} \leq\left(Q_{0} \wedge \bar{Q}_{0}\right)\right\},
$$

(cf. Theorem 5.3). They have a subtle physical meaning of redundancy. We elaborate this point.

(A) The Lüders projector $\bar{Q}$ of any class $[E]$ has no redundancy as far as occurrence goes in the sense that they occur (have probability one) in the corresponding Lüders state $\bar{\rho}$ and there exists no other event that occurs in $\bar{\rho}$ and that implies the event $\bar{Q}$.

(B) All other events $E$ that are state-dependent ly equivalent to $\bar{Q}$ do have redundancy, and a kind of its measure is uniquely expressed by the redundancy projector $\bar{E}$ because 
$E=\bar{Q}+\bar{E}$ (cf. Definition 3.5). They occur in $\bar{\rho}$, but there exists another event that also occurs in this state and it implies $E$ (it is, of course, the Lüders state).

(C) As to the events $E^{\prime}$ that are implied by $\bar{Q}$ but belong to another SD-class, one must distinguish two distinct sets of Lüders entities, besides $\bar{\rho}, \bar{Q}, \bar{Q}_{0}$ that were in focus so far, also another set $\overline{\rho^{\prime}}, \overline{Q^{\prime}}, \overline{Q_{0}^{\prime}}$ connected to the SD-class to which $E^{\prime}$ belongs.

For such events $E^{\prime}$ the redundancy projector $\overline{E^{\prime}}$ satisfies formally the same last relation $\overline{E^{\prime}} \in\left\{E^{\prime \prime}: E^{\prime \prime} \leq\left(Q_{0} \wedge \overline{Q_{0}^{\prime}}\right)\right\}$, but this time with $\overline{Q_{0}^{\prime}}$.

Next we return to restricting $D(\mathscr{\ell})$ to one of its Boolean subalgebras.

\section{SD Equivalence Classes in $B$ and Their Minimal Elements}

After having restricted $P(\mathscr{L})$ to an arbitrary Boolean subalgebra $\mathbb{B}$, "shrinking" further $\mathbb{B}$ into $B / \sim \rho$ is mathematically necessary to upgrade the preorder into an order relation (cf. Lemma 2.6). In other words, the SD implication " $\leq_{\rho}$ " is actually valid in the quotient set. Physically, the events are now the classes, and the question arises how do they "occur" or "not occur."

The subalgebra $\mathbb{B}$ can be a finite lattice, if it is closed to taking the greatest lower bound (glb) and the least upper bound (lub) of at most finite subsets; it can be a $\sigma$-lattice if it contains the glb and lub of its subsets with at most countably infinite number of elements, and, finally, it can be a complete lattice if the glb and lub of any subset is in it. We will call this the lattice type of $B$.

In restricting $P(\mathscr{H})$ to $\mathbb{B}$ each $\mathrm{SD}$ equivalence class $[E]_{\mathcal{B}}$ is part of the corresponding SD equivalence class in $D(\mathscr{\ell})$ :

$$
[E]_{\mathcal{B}} \equiv[E] \cap B
$$

The importance of the intersection relation (8.1) is in the fact that if two projectors $E, F$ are in $B$, then they belong to one and the same SD equivalence class $[E]_{B}$ if and only if they are equivalent in $P(\mathscr{H})$. For the latter, we have, besides $E \rho=F \rho$, additional necessary and sufficient conditions like in Lemma 4.6 and in Proposition 4.10.

It was seen in Corollary 5.4 that each SD equivalence class $[E]$ in $P(\mathscr{L})$ is a complete lattice. Hence, it follows from (8.1) that each SD equivalence class $[E]_{\mathcal{B}}$ in $\boldsymbol{B}$ is also a lattice of the same lattice type as that of $\boldsymbol{B}$.

To acquire a feeling for the SD equivalence classes $[E]_{B}$ defined by (8.1), we consider two extreme cases. Firstly, we assume that $\rho$ is nonsingular. Then $Q=I$ and $Q_{0}=0$. According to Remark 3.12, each projector in $P(\mathscr{\ell})$ equals the minimal element $\bar{Q}$ in its SD equivalence class. Hence all SD equivalence classes in $P(\mathscr{\ell})$ have only one element. Relation (8.1) then tells us that also the SD equivalence classes $[E]_{\mathcal{B}}$ all consist of one element and $[E]_{B}=[E]$ if $E \in B$.

Secondly, let us take a singular density operator, but let $B$ consist of only two elements. They must, of course, be 0 and $I$. Then, only $\left[Q_{0}\right](=[0])$ and $[Q](=[I])$ (cf. Lemmas 4.7 and 4.8) have nonempty intersections with $B$. The former intersection consists of only the maximal element in the $P(\mathscr{L})$ class, whereas the latter intersection contains only the minimal element of the corresponding class. We proceed by investigating the general case of $[E]_{\mathcal{B}}$ in detail.

If not stated otherwise, we assume that $B$ is a complete lattice in what follows. We introduce two key entities for further study. 
Definition 8.1. Let $Q_{0}^{\mathcal{B}}$ be the greatest subprojector of $Q_{0}$ that is in $\mathbb{B}: Q_{0}^{\mathcal{B}} \equiv \vee\left(\left\{E: E \leq Q_{0}\right\} \cap \mathbb{B}\right)$. We call $Q_{0}^{\mathcal{B}}$ the Boolean initial null projector. Further, let $Q_{\mathcal{B}}$ be the least among the projectors that are greater than $Q$ and belong to $\mathbb{B}: Q_{\mathcal{B}} \equiv \wedge(\{E: E \geq Q\} \cap B)$. We call $Q_{\mathcal{B}}$ the Boolean initial range projector.

Note that, since $B,\left\{E: E \leq Q_{0}\right\}$, and $\{E: E \geq Q\}$ are complete lattices (cf. Lemma 5.2 and its proof in Appendix $C$ ), so are the above intersections.

Proposition 8.2. The Boolean null projector and the Boolean range projector are dual entities, that is, they are the ortho-complementary projectors of each other: $Q_{0}^{\mathcal{B}}=Q_{\bar{B}}^{c}$.

Proof. The SD equivalence classes $\left\{E: E \leq Q_{0}\right\} \cap B$ and $\{E: E \geq Q\} \cap B$ are dual to each other. Hence, so are the maximal element in the former and the minimal element in the latter.

Remark 8.3. Evidently, $Q_{\mathcal{B}}=Q$, or equivalently $Q_{0}^{\mathcal{B}}=Q_{0}$ if and only if $Q \in \mathbb{B}$, or equivalently $Q_{0} \in ß$.

Proposition 8.4. One has $[0]_{\mathcal{B}}=\left[Q_{0}^{\mathcal{B}}\right]_{\mathcal{B}}$ and $\left[Q_{\mathcal{B}}\right]_{\mathcal{B}}=[I]_{\mathcal{B}}$. The zero projector and $Q_{0}^{\mathcal{B}}$ are the least and the greatest elements, respectively, in $[0]_{\mathcal{B}}: E \in[0]_{\mathcal{B}} \Rightarrow 0 \leq E \leq Q_{0}^{B}$, whereas $Q_{B}$ and the identity operator $I$ are the least and the greatest elements, respectively, in $[I]_{B}: E \in[I]_{B} \Rightarrow Q_{B} \leq E \leq I$.

Proof. The projector $Q_{0}^{\mathcal{B}}$ is by Definition 8.1 a subprojector of $Q_{0}$. Hence, $Q_{0}^{\mathcal{B}} \rho=0 \rho$, or $Q_{0}^{\mathcal{B}} \sim \rho_{0} 0$. The projector $Q_{0}^{B}$ is, by definition, the greatest subprojector of $Q_{0}$ that belongs to $B$. Hence it is the greatest element in $[0]_{\mathcal{B}}$. Further, as we know (cf. lemma 2 and lemma 3), one has $E \rho=\rho$, or equivalently $E \sim_{\rho} I$, if and only if $E \geq Q$. Hence, $Q_{\mathcal{B}}$ is the minimal element of $[I]_{\mathcal{B}}$. The rest is obvious.

Definition 8.5. We call a projector $E_{0}^{\mathcal{B}}$ a core element in $\mathcal{B}$ if the relation $E_{0}^{\mathcal{B}} \mathrm{Q}_{0}^{\mathcal{B}}=0$ is satisfied. The set of all core elements in $B$ we call the core set and denote it by $C_{B}$.

Proposition 8.6. The core set is $C_{B}=\left\{E: E \leq Q_{B}\right\} \cap B$. It is a complete lattice.

Proof. The definition $E_{0}^{\mathcal{B}} Q_{0}^{\mathcal{B}}=0$ means that each core element is orthogonal to $Q_{0}^{\mathcal{B}}$, or equivalently that it is a subprojector of the ortho-complementary projector $Q_{\mathcal{B}}$ of $Q_{0}^{\bar{B}}$ (cf. Proposition 8.2). The poset $C_{B}$ is the intersection of two complete lattices, hence it is itself a complete lattice

Theorem 8.7. (A) Every state-dependent equivalence class $[E]_{\mathcal{B}}$ contains a core element $E_{0}^{\mathcal{B}}$. It can be evaluated from an arbitrary element $E$ of the class by the relation

$$
E_{0}^{\mathcal{B}}=E-E Q_{0}^{\mathcal{B}}
$$

(cf. Definitions 8.1 and 8.5).

(B) Every core element $E_{0}^{\mathcal{B}}$ is the least element of its class $\left[E_{0}^{\mathcal{B}}\right]_{\mathcal{B}}: E \sim{ }_{\rho} E_{0}^{\mathcal{B}}, E \in \mathcal{B} \Rightarrow E \geq$ $E_{0}^{\mathcal{B}}$. Hence, each class contains a unique core element.

Proof. (A) Let $[E]_{\mathcal{B}}$ be any state-dependent equivalence class in $B$, and let $E$ be any of its elements. The rhs of (8.2) is state-dependently equivalent to $E$ because $Q_{0}^{\mathcal{B}}$ is equivalent to 0 . In other words, the lhs belongs to $[E]_{\mathcal{B}}$. We prove that it is a core element: $\left(E-E Q_{0}^{\mathcal{B}}\right) Q_{0}^{\mathcal{B}}=0$. 
Hence, according to Definition 8.5, it is a core element, and therefore, each class does contain at least one core element.

(B) Let $E_{0}^{\mathcal{B}}$ be a core element of $[E]_{\mathcal{B}}$. Then, according to Theorem 8.7(A), $E_{0}^{\mathcal{B}}=E-E Q_{0}^{\mathcal{B}}$. Multiplication gives $E E_{0}^{\mathcal{B}}=E_{0}^{\mathcal{B}}$, that is, $E_{0}^{\mathcal{B}} \leq E$ as claimed. This makes $E_{0}^{\mathcal{B}}$ the unique least element in the class $\left[E_{0}^{\mathcal{B}}\right]_{\mathcal{B}}$ to which it belongs.

The core elements $E_{0}^{\mathcal{B}}$ in the SD equivalence classes in $\mathcal{B}$ play the same role as the Lüders range projectors $\bar{Q}$ in the SD equivalence classes in $P(\mathscr{L})$ (cf. Corollary 5.4).

\section{Further Investigation of the SD Equivalence Classes in $B$}

Some SD equivalence classes $[E]$ in $p(\mathscr{H})$ do and others do not contain an SD equivalence class $[E]_{\mathcal{B}}$, that is, they may be vacant in this respect. We now derive some necessary conditions for nonvacant classes $[E]$.

Proposition 9.1. Each $S D$ equivalence class $[E]$ is vacant unless $Q_{B}$ is an upper bound for the minimal element, the Lüders range projector $\bar{Q}$, in the class.

Proof. The core element $E_{0}^{\mathcal{B}}$, if it exists in a class $\left[E_{0}^{\mathcal{B}}\right]$, must be an upper bound of the minimal element $\bar{Q}$ in the class. Since, further, $E_{0}^{\mathcal{B}} \leq Q_{\mathcal{B}}$ (cf. Proposition 8.6), transitivity establishes the claim.

Corollary 9.2. In the special case when $Q_{\mathcal{B}}=Q$, or equivalently, $Q \in \mathbb{B}, S D$ equivalence classes in $\mathbb{B}$ can appear only in those $S D$ equivalence classes in $P(\mathscr{H})$ in which $\bar{Q} \leq Q$, and then not necessarily in all (take, e.g., a non-singular $\rho$ and the minimal Boolean subalgebra).

We now investigate the properties of the intermediate elements of the SD equivalence classes in $\mathbb{B}$ analogously as one did in $P(\mathscr{L})$.

Theorem 9.3. (A) Each element $E$ of each state-dependent equivalence class $[E]_{B}$ can (uniquely) be written as follows:

$$
E=E_{0}^{\mathcal{B}}+E^{\prime}
$$

so that

$$
E_{0}^{\mathcal{B}} \sim \rho E, \quad E_{0}^{\mathcal{B}} \in C_{\bar{B}}, \quad E^{\prime} \in[0]_{\mathcal{B}} .
$$

Conversely, for each core element $E_{0}^{\mathcal{B}}$, each projector $E^{\prime} \in[0]_{\mathcal{B}}$ determines an element $E \equiv$ $E_{0}^{\mathcal{B}}+E^{\prime}$ that is state-dependently equivalent to $E_{0}^{\mathcal{B}}$. In this way one has a one-to-one map of $\left\{E^{\prime}: E^{\prime} \leq\right.$ $\left.Q_{0}^{\mathcal{B}}\right\} \cap B\left(=[0]_{\mathcal{B}}=\left[Q_{0}^{\mathcal{B}}\right]_{\mathcal{B}}\right)$ onto any class $\left[E_{0}^{\mathcal{B}}\right]_{\mathcal{B}}$.

(B) The map associating with each $E^{\prime} \in[0]_{\mathcal{B}}$ the image $E_{0}^{\mathcal{B}}+E^{\prime}$, where $E_{0}^{\mathcal{B}}$ is the core element of any given $S D$ equivalence class in $B$, is an isomorphism of the poset $[0]_{B}$ onto the poset $\left[E_{0}^{B}\right]_{B}$.

Proof. (A) Theorem 8.7 has established that each SD equivalence class $[E]_{\mathcal{B}}$ contains a unique core element $E_{0}^{\mathcal{B}}$ and that the latter is the least element of the class. The projector $E^{\prime} \equiv E-E_{0}^{\mathcal{B}}$ is obviously in $\mathbb{B}$ since one can write $E^{\prime}=\left(E_{0}^{\mathcal{B}}\right)^{\mathcal{C}} E$. That $E^{\prime}$ is a subprojector of $Q_{0}^{\mathcal{B}}$ is seen as 
follows. We take into account that $E_{0}^{\mathcal{B}} \rho=E \rho$, which implies $E^{\prime} \rho=0, E^{\prime} \sim_{\rho} Q_{0}^{\mathcal{B}}$ and $E^{\prime} \leq Q_{0}^{\mathcal{B}}$ (cf. Proposition 8.4). Thus, $E^{\prime} \in[0]_{B}$.

Conversely, we assume that

$$
E^{\prime} \leq Q_{0}^{B}, \quad E^{\prime} \in \mathbb{B} .
$$

One has $E_{0}^{\mathcal{B}} \leq Q_{\mathcal{B}}$ (cf. Definition 8.5 and Proposition 8.4), and, taking the ortho-complement of this inequality, $Q_{0}^{\mathcal{B}} \leq\left(E_{0}^{\mathcal{B}}\right)^{c}$ ensues. Hence, transitivity and (9.3) give $E^{\prime} \leq\left(E_{0}^{\mathcal{B}}\right)^{c}$, implying $E^{\prime} E_{0}^{\mathcal{B}}=0$. This orthogonality makes it possible to add $E^{\prime}$ to $E_{0}^{\mathcal{B}}$.

Finally, as we know from Proposition 8.4, $E^{\prime} \rho=0$. Hence, $\left(E_{0}^{\mathcal{B}}+E^{\prime}\right) \sim{ }_{\rho} E_{0}^{\mathcal{B}}$ as claimed. The map at issue is clearly a one-to-one map because it can be inverted beginning with any element of the image set $[E]_{\mathcal{B}}$.

(B) To prove isomorphism, let $E^{\prime} \leq E^{\prime \prime}, E^{\prime}, E^{\prime \prime} \in[0]_{B}$. Let us, further, multiply out the product

$$
\left(E_{0}^{\mathcal{B}}+E^{\prime}\right)\left(E_{0}^{\mathcal{B}}+E^{\prime \prime}\right)=E_{0}^{\mathcal{B}}+E_{0}^{\mathcal{B}} E^{\prime \prime}+E^{\prime} E_{0}^{\mathcal{B}}+E^{\prime} E^{\prime \prime}
$$

One obtains

$$
\left(E_{0}^{\mathcal{B}}+E^{\prime}\right)\left(E_{0}^{\mathcal{B}}+E^{\prime \prime}\right)=E_{0}^{\mathcal{B}}+E^{\prime} E^{\prime \prime}
$$

if one utilizes the orthogonalities stemming from $E_{0}^{\mathcal{B}} E^{\prime \prime}=E_{0}^{\mathcal{B}}\left(Q_{0}^{\mathcal{B}} E^{\prime \prime}\right)=0$ (cf. Definition 8.5), and $E^{\prime} E_{0}^{\mathcal{B}}=\left(E^{\prime} Q_{0}^{\mathcal{B}}\right) E_{0}^{\mathcal{B}}=0$. Obviously, if and only if $E^{\prime} E^{\prime \prime}=E^{\prime}$, then $\left(E_{0}^{\mathcal{B}}+E^{\prime}\right)\left(E_{0}^{\mathcal{B}}+E^{\prime \prime}\right)=E_{0}^{\mathcal{B}}+E^{\prime}$ as claimed.

Corollary 9.4. Each $S D$ equivalence class $[E]_{B}$ has, as a poset, the same structure as the poset $\left[Q_{0}^{\mathcal{B}}\right]_{\bar{B}}$. In particular, in the former, its core element $E_{0}^{\mathcal{B}}$ is the least and $E_{0}^{\mathcal{B}}+Q_{0}^{\mathcal{B}}$ is the greatest element.

Corollary 9.5. Each $S D$ equivalence class $[E]_{B}$ has precisely one element, the core element $E_{0}^{\mathcal{B}}$, if and only if $Q_{0}^{B}=0$. This is the case, for example, when $\rho$ is non-singular, because then $Q_{0}=0$, and $Q_{0}^{\mathcal{B}} \leq Q_{0}$. But $Q_{0}$ may be non-zero, and $Q_{0}^{\mathcal{B}}=0$.

Corollary 9.6. Theorem 9.3 establishes an isomorphism between any two SD equivalence classes in B (in an obvious way).

The reader who remembers well the results in the decomposition of entire quantum $\operatorname{logic} P(\mathscr{\ell})$ into SD equivalence classes is rightly perplexed. He wonders how did it happen that restriction to a Boolean subalgebra made all the $S D$ equivalence classes $[E]_{B}$ mutually isomorphic, whereas the larger SD equivalence classes [E] (cf. (1.1)) are not isomorphic due to the fact that the Lüders null projectors $\bar{Q}_{0}$ are, in general, different for different classes.

To throw additional light on this puzzling fact, we shortly repeat the relevant parts of the above arguments. The (minimal) core elements $E_{0}^{\mathcal{B}}$ in the classes $[E]_{\mathcal{B}}$ are obtained from an arbitrary element in the class in a completely parallel way as in $[E]$ (cf. Theorem 8.7(A) above and Theorem 3.7). As a consequence, the core elements satisfy $E_{0}^{\mathcal{B}} Q_{0}^{\mathcal{B}}=0$ parallelly to the Lüders range projectors for which $\bar{Q} \wedge Q_{0}=0$ is valid (cf. Definition 8.5 above and Corollary 3.9). 
Also Theorem 9.3(A) above could have been put in the form of a map of $\left\{E^{\prime}: E^{\prime} \leq\right.$ $\left.Q_{0}^{\mathcal{B}} \wedge\left(E_{0}^{\mathcal{B}}\right)^{\mathcal{C}}\right\} \cap \mathcal{B}$ (see below) onto $[E]_{\mathcal{B}}$, to display further parallelism with $\mathcal{D}(\mathcal{H})$, where the counterpart is $\left\{E^{\prime} \leq Q_{0} \wedge \bar{Q}^{c}\right\}$. So far the classes in $B$ and in $P(\mathscr{C})$ go parallelly.

What makes a difference is the fact that in a Boolean subalgebra any two projectors commute. Hence, glb takes the form of multiplication. If the glb is zero, as it is in our case (cf. Definition 8.5), we have orthogonality, leading to $E_{0}^{\mathcal{B}} \leq Q_{\mathcal{B}}$ and, taking the ortho-complement of this inequality, to $Q_{0}^{\mathcal{B}} \leq\left(E_{0}^{\mathcal{B}}\right)^{c}$. Since in a Boolean subalgebra the last inequality means $Q_{0}^{\mathcal{B}}\left(E_{0}^{\mathcal{B}}\right)^{\mathcal{C}}=Q_{0}^{\mathcal{B}}$, we have $Q_{0}^{\mathcal{B}} \wedge\left(E_{0}^{\mathcal{B}}\right)^{\mathcal{C}}=Q_{0}^{\mathcal{B}}\left(E_{0}^{\mathcal{B}}\right)^{\mathcal{C}}=Q_{0}^{\mathcal{B}}$ as a consequence.

Thus, $\left(E_{0}^{\mathcal{B}}\right)^{c}$, the counterpart of $\bar{Q}_{0}$, has disappeared from the scene (whereas $\bar{Q}_{0}\left(=\bar{Q}^{c}\right.$ ) in $[E]$ does remain in general).

Remark 9.7. If $\bar{Q} \leq Q$, then, taking the ortho-complementary projectors, we obtain $Q_{0} \leq \bar{Q}_{0}$ implying $Q_{0} \wedge \bar{Q}_{0}=Q_{0}$. In other words, in the classes considered, $\bar{Q}_{0}$ disappears from the maximal element in the class, which is now $\bar{Q}+Q_{0}$. Thus, the considered classes are mutually isomorphic just like the SD equivalence classes in $B$ that they may contain.

\section{The Core Decomposition}

The core set $\mathcal{C}_{B}$ in $B$ (cf. Definition 8.5) runs parallel to the Lüders set $\mathcal{L}$ in $P(\mathscr{L}$ ) (cf. Proposition 5.1(C)). We investigate now if also the lattice structure of the former parallels that of $\mathcal{L}$.

Theorem 10.1. The core set $\mathcal{C}_{B}$ has the structure of a complete lattice with the greatest lower bound operation being the same as in $\mathrm{B}$, but the least upper bound operation being, in general, different. The least element in $\mathcal{C}_{B}$ is the zero projector 0 , and the greatest element is $Q_{B}$.

Proof. Let $\mathcal{S}$ be any subset of $\mathcal{C}_{B}$, and let $\wedge \mathcal{S}$ be the glb of the projectors in $\mathcal{S}$ taken in $P(\mathscr{\ell})$. We show that it is a core element.

Arguing ab contrario, we assume that there exists a non-zero projector $E$ in $B$ that is a common subprojector of $\wedge \mathcal{S}$ and of $Q_{0}^{B}$. But then, on account of $\wedge \mathcal{S}$ being a lower bound (a subprojector) of each $E_{0}^{\mathcal{B}} \in \mathcal{S}$ and of $Q_{0}^{\mathcal{B}}$, it cannot be non-zero (cf. Definition 8.5), and $\wedge \mathcal{S} \in \mathcal{C}_{B}$ follows. It is obvious that zero is the least element in $\mathcal{C}_{B}$.

The projector $Q_{\mathcal{B}}$ is a core element (cf. Definition 8.5) since it is orthogonal to $Q_{0}^{\mathcal{B}}$ as its ortho-complementary projector. That it is the largest element in $\mathcal{C}_{B}$ is evident from Proposition 8.6.

It is an elementary fact that every complete glb-semilattice with a greatest element is a complete lattice because the lub of any subset is the glb of the subset of all upper bounds of the given subset (for the reader's convenience the latter claim is proved in Appendix D).

The lub $\vee \mathcal{S}$ in $\mathcal{C}_{B}$ need not be the same as the lub of the same subset in $\mathbb{B}$, in general, because, in the latter case, it equals the glb of the subset of all upper bounds of the given subset in $\mathbb{B}$. This is the glb of a larger set, hence it is, in general, less than the mentioned glb in $\mathcal{C}_{B}$.

Unlike in $P(\mathscr{\ell})$, the core set has isomorphic complete lattices.

Theorem 10.2. For each $E^{\prime} \in[0]_{\mathcal{B}}\left(=\left[Q_{0}^{B}\right]_{B}\right)$, the poset $E^{\prime}+\mathcal{C}_{B}$, which we call the core class determined by $E^{\prime}$, with the absolute implication $\leq$ defined in $D(\mathscr{d})$, is isomorphic to $\mathcal{C}_{B}$; hence, it is a complete lattice like $\mathcal{C}_{B}$. 
One should note that $E^{\prime}+\mathcal{C}_{B} \equiv\left\{E^{\prime}+E: E \in \mathcal{C}_{B}\right\}$ is the set of all projector sums of $E^{\prime}$ and an element of $C_{B}$. A sum of two projectors is a projector if the terms are orthogonal as well known. It is the case here because $E^{\prime} \leq Q_{0}^{\mathcal{B}}$ (cf. Theorem 9.3(A) and Proposition 8.4), $E_{0}^{\mathcal{B}} \leq Q_{\mathcal{B}}$ (cf. Proposition 8.6), and $Q_{0}^{\mathcal{B}}$ and $Q_{\mathcal{B}}$ are orthogonal to each other (cf. Proposition 8.2).

Proof of Theorem 10.2. Let $E^{\prime} \in[0]_{\mathcal{B}}, E_{0}^{\mathcal{B}}, E_{0}^{\prime B} \in \mathcal{C}_{B}$. On account of the orthogonalities due to Definition 8.5 and the fact that $E^{\prime} \leq Q_{0}^{B}$, one has

$$
\left(E^{\prime}+E_{0}^{\mathcal{B}}\right)\left(E^{\prime}+E_{0}^{\prime \mathcal{B}}\right)=E^{\prime}+E_{0}^{\mathcal{B}} E_{0}^{\prime \mathcal{B}} .
$$

This is $\left(E^{\prime}+E_{0}^{\mathcal{B}}\right)\left(E^{\prime}+E_{0}^{\prime \mathcal{B}}\right)=E^{\prime}+E_{0}^{\mathcal{B}}$, that is, $\left(E^{\prime}+E_{0}^{\mathcal{B}}\right) \leq\left(E^{\prime}+E_{0}^{\prime \mathcal{B}}\right)$ if and only if $E_{0}^{\mathcal{B}} E_{0}^{\prime \mathcal{B}}=E_{0}^{\mathcal{B}}$, that is, $E_{0}^{\mathcal{B}} \leq E_{0}^{\prime \mathcal{B}}$.

Definition 10.3. We call the (nonoverlapping) decomposition of $\mathbb{B}$

$$
B=\sum_{E^{\prime} \in[0]_{B}}\left\{E^{\prime}+\mathcal{C}_{B}\right\}
$$

the core decomposition of the given Boolean subalgebra $\mathbb{B}$ of $D(\mathscr{\ell})$ into core classes.

We can picture $B$ as a matrix of elements such that the rows are the SD equivalence classes and the columns are the core classes. Then, the rows are enumerated by the core elements (elements of $\mathcal{C}_{B}$ ), and the columns by the elements of $[0]_{B}$.

One should pay attention to the fact that the same notation is used for summation (+ and $\Sigma$ ) in two different senses: as a summation of projectors like in the core class $E^{\prime}+\mathcal{C}_{B}$, and as a union of non-overlapping subsets as in Definition 10.3.

Remark 10.4. In view of Remark 9.7, we see that if we restrict ourselves to $\mathcal{N V} \equiv \sum_{\bar{Q} \leq Q}[\bar{Q}]$ (a subset of $P(\mathscr{L})$ ), that is, if we consider only those SD equivalence classes in $P(\mathscr{L})$ that may contain a SD equivalence class in $\mathcal{B}$ (that are nonvacant in this respect), then we have a decomposition of $\mathcal{N U}$ parallelling the core decomposition of $B$. But in $P(\mathscr{L})$, when no $B$ is chosen, there is no motivation for this.

\section{Back to the SD Quotient Set}

There is more insight to be gained about the poset structure of the SD quotient set $B / \sim \rho$.

A remarkable property of $B / \sim_{\rho}$ is the following fact [11] (Lemma 1 there). A SD equivalence class implies state-dependently another such class if and only if there is an event $E$ in the former and an event $F$ in the latter so that $E \leq F$, that is, the former event implies the latter one in the absolute sense. Since this result is of great importance for the aims of this study, we re derive it (with new insight) to make the present text self-contained.

Proposition 11.1. If $E \leq F$, then $[E]_{B} \leq_{\rho}[F]_{B}$. Conversely, if $[E]_{B} \leq_{\rho}[F]_{B}$, then $\exists E^{\prime} \in[E]_{B}$ and $F^{\prime} \in[F]_{B}$ such that $E^{\prime} \leq F^{\prime}$.

Proof. The relation $E \leq F$ can be rewritten as $E \leq_{\rho} F$ (cf. Corollary 2.3), then the claimed relation $[E]_{B} \leq_{\rho}[F]_{B}$ immediately follows (cf. Definition 2.5). If $[E]_{B} \leq_{\rho}[F]_{B}$, then $E \rho=E F \rho$ 
(cf. Propositions 2.4 and 2.1). Hence, $E F \sim_{\rho} E$, that is, $E F \in[E]_{\mathcal{B}}$, and, taking $E^{\prime} \equiv E F$ and $F^{\prime} \equiv F$ one has $E^{\prime} \leq F^{\prime}$.

Remark 11.2. In the partially ordered set $B / \sim_{\rho}$ one can replace $\leq_{\rho}$ by $\leq$ because Proposition 11.1 allows one to define $[E]_{\mathcal{B}} \leq[F]_{\mathcal{B}}$ via $E \leq F$ that the respective $C D$ equivalence classes contain.

For the sake of generality, let us assume now that the Boolean subalgebra $B$ is of any lattice type (finite, $\sigma$ - or complete, cf. the second passage in section 8 ).

Theorem 11.3. (A) The map associating with each projector $E \in \mathbb{B}$ the $S D$ equivalence class $[E]_{B}$ to which it belongs is a poset homomorphism of the Boolean subalgebra $B$ onto the Boolean algebra $B / \sim_{\rho}$.

(B) $B / \sim \rho$ is of the same lattice type as $B$.

Proof. (A) In view of Proposition 11.1, it is obvious that the map taking $\mathbb{B}$ onto $\mathbb{B} / \sim_{\rho}$ by associating with each element $E \in \mathbb{B}$ the SD equivalence class $[E]_{\mathcal{B}}$ to which it belongs is a homomorphism of a poset with absolute implication " $\leq$ " onto the poset $B / \sim \rho$ with the same implication " $\leq$." The map evidently preserves the ortho-complementation operation. We prove now that it preserves also the glb and lub operations.

Let $\left\{E_{m}: m \in M\right\}$ be an arbitrary subset of $\mathcal{B}$ of a finite number of elements if $\mathcal{B}$ is a finite lattice, of at most a countably infinite number of elements if $\mathcal{B}$ is a $\sigma$-lattice, and a completely arbitrary subset if $B$ is a complete lattice.

The SD equivalence class $\left[\wedge_{m \in M} E_{m}\right]_{B}$ is a lower bound of the set $\left\{\left[E_{m}\right]_{B}: m \in M\right\}$ of classes because so is $\wedge_{m \in M} E_{m}$ for the set of projectors $\left\{E_{m}: m \in M\right\}$. If $[F]_{B}$ is an arbitrary lower bound of $\left\{\left[E_{m}\right]_{\mathcal{B}}: m \in M\right\}$, then $\forall m \in M: \exists F_{m} \in[F]_{\mathcal{B}}$ such that $F_{m} \leq E_{m}$ (cf. Proposition 11.1 and its proof). Let $F^{\prime} \equiv \wedge_{m \in M} F_{m}$. Since $\forall m \in M: F^{\prime} \leq F_{m} \leq E_{m}$, one has $F^{\prime} \leq \wedge_{m \in M} E_{m}$.

On the other hand, $F^{\prime} \in[F]_{B}$ because also $[F]_{B}$ is a lattice of the specified lattice type (cf. (8.1)). Hence, on account of the last inequality, $[F]_{\mathcal{B}} \leq\left[\wedge_{m \in M} E_{m}\right]_{\mathcal{B}}$, and $\left[\wedge_{m \in M} E_{m}\right]_{\mathcal{B}}$ is seen to be the glb of $\left\{\left[E_{m}\right]_{B}: m \in M\right\}$. Thus the map in question preserves the glb operation. Symmetrically (or dually) one can prove preservation of the lub operation.

To prove that the quotient set is an ortho-complemented lattice, let again $\left\{\left[E_{m}\right]_{B}: m \in\right.$ $M$ \} be an arbitrary subset of $\boldsymbol{B}$ (of cardinality corresponding to the lattice type of $\boldsymbol{B}$ ). Since the first Morgan rule $\left(\wedge_{m \in M} E_{m}\right)^{c}=\vee_{m \in M} E_{m}^{c}$ is valid in $\mathbb{B}$, and the surjection is such that $[E]_{\mathcal{B}}^{c}=\left[E^{c}\right]_{\mathcal{B}}$, one obtains the validity of the first Morgan rule $\left.\left(\wedge_{m \in M}\left[E_{m}\right]_{\mathcal{B}}\right)^{c}=\vee_{m \in M}\left[E_{m}\right]_{\mathcal{B}}^{c}\right)$ in $B / \sim \rho$. Symmetrically one proves that also the second Morgan rule is true.

To show that $B / \sim \rho$ is a Boolean algebra, we have to prove that the glb and the lub operations are mutually distributive. It is, actually, a straightforward consequence of the preservation of these operations in the map $E \rightarrow[E]_{B}$ :

$$
[E]_{B} \wedge\left([F]_{B} \vee[G]_{B}\right)=[E]_{B} \wedge[F \vee G]_{B}=[E \wedge(F \vee G)]_{B}
$$

Since $\mathbb{B}$ is a Boolean subalgebra, it is distributive, and one further has

$$
\begin{aligned}
\text { lhs }=[(E \wedge F) \vee(E \wedge G)]_{\mathcal{B}} & =[(E \wedge F)]_{\mathcal{B}} \vee[(E \wedge G)]_{\mathcal{B}} \\
& =\left([E]_{\mathcal{B}} \wedge[F]_{\mathcal{B}}\right) \vee\left([E]_{\mathcal{B}} \wedge[G]_{\mathcal{B}}\right) .
\end{aligned}
$$

The converse distributivity of lub with respect to glb is proved analogously. 
(B) We prove now that $\mathcal{B} / \sim_{\rho}$ is a lattice of the same lattice type as $\mathcal{B}$. Let $\left\{\left[E_{m}\right]_{\mathcal{B}}: m \in\right.$ $M$ \} be an arbitrary subset of the former (of cardinality corresponding to the lattice type of $B$ ). Then $\wedge_{m \in M} E_{m}$, being the glb of $\left\{E_{m}: m \in M\right\}$, makes (according to the preceding passage) $\left[\wedge_{m \in M} E_{m}\right]_{\mathcal{B}}$ the glb of $\left\{\left[E_{m}\right]_{B}: m \in M\right\}$. Symmetrically one deals with the lub operation.

The results of this and the next section, in conjunction with all the results of the four articles on the topic ([11-13], and this paper) make it, hopefully, desirable to accept the proposed term "SD quantum logic" in its two meanings (cf. Remark 2.7).

\section{Physical Meaning of the Quotient Set}

We have seen that SD implication extends absolute implication in a given Boolean subalgebra $B$ of quantum logic $D(\mathscr{\ell})$, but there is a price to be paid: SD implication is a preorder. Inherent in it is an equivalence relation that makes equivalent events indistinguishable. These are the events that have same probability in the given state $\rho$, and change this state in the same way when ideally measured.

When one makes the transition to the SD quotient set $B / \sim_{\rho}$ (by a homomorphism of a Boolean subalgebra to a Boolean algebra of the same kind, cf. Theorem 11.3), then one can even forget about the SD implication, because $B / \sim_{\rho}$ can be characterized by the equivalence relation in $\bar{B}$ and absolute implication (cf. Remark 11.2).

This throws new light on SD implication. The SD quotient set, that is, the SD quantum logic (cf. Remark 2.7), can be viewed, instead of as extending absolute implication in $B$, as a contraction, a shrinking of sets of indistinguishable events with respect to $\rho$ into, effectively, single events (the corresponding SD equivalence classes in $\mathbb{B}$ ).

This is where, when $B$ is a complete lattice, the extreme elements of the SD equivalence classes acquire physical meaning. As it has been shown in Propositions 6.1 and 6.2 (cf. also Corollary 9.4), occurrence of a SD equivalence class amounts to the occurrence of its minimal element, the core element $E_{0}^{\mathcal{B}}$, and its non-occurrence to that of its maximal element $E_{0}^{\mathcal{B}}+Q_{0}^{\mathcal{B}}$.

If for a set of SD equivalence classes, that is, elements of the SD quantum logic $B / \sim \rho$, the question of occurrence or non-occurrence appears, then the physical answer is given by the corresponding glb and lub operation in the core set $\mathcal{C}_{B}$ (cf. Definition 8.5 and Proposition 8.6).

\section{An Illustration}

Let the state space be a separable, that is, a countably infinite dimensional, complex Hilbert space $\mathscr{H}$, and let $\{|n\rangle: n=1,2, \ldots, \infty\}$ be a given complete orthonormal basis in $\mathscr{H}$. We consider an observable $A$ defined by its spectral form

$$
A=\sum_{n} a_{n}|n\rangle\langle n|, \quad n \neq n^{\prime} \Longrightarrow a_{n} \neq a_{n^{\prime}}
$$

and a state vector

$$
|\Psi\rangle \equiv \sum_{n=2,4, \ldots, \infty} \psi_{n}|n\rangle ; \quad \sum_{n=2,4, \ldots, \infty}\left|\psi_{n}\right|^{2}=1
$$

The last two sums are over all even integers, and all $\psi_{n}$ in them are non-zero. 
We define the Boolean subalgebra $B$ as the set of all spectral projectors of the observable $A$. Let $\mathcal{S}$ be an arbitrary set of integers including the vacant set, and let $\mathcal{S}=\mathcal{S}^{e}+\mathcal{S}^{\circ}$ be the unique decomposition of $\mathcal{S}$ into the subset of all even integers that it contains and the subset of all odd integers in it. Let us denote by $D$ the power set, that is, the set of all subsets (the vacant set included) of the set $\mathcal{N}$ of all integers.

It is easily seen that $P$ can be used as the index set of the spectral projectors, that is, that $B \equiv\left\{E_{\mathcal{S}}: \mathcal{S} \in P\right\}, E_{\mathcal{S}} \equiv \sum_{n \in \mathcal{S}}|n\rangle\langle n| . B$ and $\boldsymbol{D}$ are isomorphic posets with respect to the map $D \ni \mathcal{S} \rightarrow E_{\mathcal{S}} \in \mathcal{B}$. In $B$ absolute implication " $\leq$ " defined in $P(\mathscr{H})$, and in $D$ the set-theoretical inclusion relation " $\subseteq$ " are the partial orders. Evidently, $D$ and $B$ are complete lattices.

The SD equivalence classes are defined by the SD equivalence relation

$$
E_{\mathcal{S} \sim \Psi\rangle} E_{\mathcal{S}^{\prime}} \Longleftrightarrow E_{\mathcal{S}}|\Psi\rangle=E_{\mathcal{S}^{\prime}}|\Psi\rangle
$$

Evidently, $E_{\mathcal{S}} \sim{ }_{\rho} E_{\mathcal{S}^{\prime}}$ if and only if $\mathcal{S}^{e}=\left(\mathcal{S}^{\prime}\right)^{e}$. Hence. the SD equivalence classes are the sets $\left\{E_{\mathcal{S}}: \forall \mathcal{S}^{o}, \mathcal{S}^{e}\right.$ fixed $\}$ with $E_{\mathcal{S} e}$ as the core elements.

To find the greatest elements in the SD equivalence classes, we note that $E_{\mathcal{S}_{M}} \equiv$ $\sum_{n=1,3, \ldots, \infty}|n\rangle\langle n|$, where the summation goes over all odd integers, is the greatest spectral projector that takes $|\Psi\rangle$ into zero. It is the Boolean null projector $Q_{0}^{\mathcal{B}}=E_{\mathcal{S}_{M}}$. The greatest elements in the $\mathrm{SD}$ equivalence classes are $E_{\mathcal{S}_{e}}+E_{\mathcal{S}_{M}}$. The Boolean range projector is $Q_{B}=$ $\left(Q_{0}^{B}\right)^{c}=E_{\mathcal{S}_{M}}^{c}=\sum_{n=2,4, \ldots, \infty}|n\rangle\langle n|$, where the summation goes over all even integers.

In particular, the projectors 0 and $E_{\mathcal{S}_{M}}$ are the extreme elements of $[E]_{B}$ when $E|\Psi\rangle=0$, and $E_{\mathcal{S}_{M}}^{c}$ and $I$ are the extreme elements of $[E]_{B}$ if $E \rho=\rho$ (cf. Proposition 8.4).

Incidentally, the initial range projector is $Q=|\Psi\rangle\langle\Psi|$, and the initial null projector is $Q_{0}=I-|\Psi\rangle\langle\Psi|$. In a given SD equivalence class $\left[E_{0}^{\mathcal{B}} \equiv E_{\mathcal{S}^{e}}\right]$ in $\mathcal{D}(\mathscr{H})$ the Lüders range projector is $\bar{Q}=E_{0}^{\mathcal{B}}-\left(E_{0}^{\mathcal{B}} \wedge Q_{0}\right)$.

The core set is $\mathcal{C}_{B}=\left\{E_{\mathcal{S}^{e}}: \forall \mathcal{S}^{e}\right\}$. The core classes are

$$
\left\{E_{\mathcal{S}^{\circ}}+E_{\mathcal{S}^{e}}: \forall E_{\mathcal{S}^{e}}, E_{\mathcal{S}^{e}} \in \mathcal{C}_{B} ; E_{\mathcal{S}^{\circ}} \text { fixed }\right\} .
$$

Thus an arbitrary element $E$ in $\mathcal{B}$ is uniquely decomposed as $E=E_{0}^{\mathcal{B}}+E^{\prime}$, where $E_{0}^{\mathcal{B}} \equiv E_{\mathcal{S}^{e}}$, and $E^{\prime} \equiv E_{\mathcal{S}^{\circ}}$. If one pictures $\boldsymbol{B}$ as a matrix of elements (projectors) so that the SD equivalence classes are the rows and the core classes are the columns, then $E_{0}^{\mathcal{B}}$ shows in which row, and $E^{\prime}$ displays in which column the element is.

One could replace the above density operator $|\Psi\rangle\langle\Psi|$ by a mixed state, and make the observable incomplete with arbitrary multiplicities of the eigenvalues, but still with a purely discrete spectrum. Such an observable would be the general one that is measured exactly. But the additional intricacies would then diminish the transparency of the illustration.

\section{Appendices}

\section{A. Proof of Lemma 3.1}

Claiming the general validity of the equivalence relation

$$
\operatorname{tr}(E \rho)=1 \Longleftrightarrow Q \leq E
$$

where $Q$ is the range projector of $\rho$. 
Let $\{|i\rangle: \forall i\}$ be an eigen-basis of $\rho$ spanning its range, that is, let $Q=\sum_{i}|i\rangle\langle i|$ and $\rho=\sum_{i} r_{i}|i\rangle\langle i|$, where $\left\{r_{i}: \forall i\right\}$ is the positive spectrum of $\rho$. Then $1=\operatorname{tr}(E \rho)$ is equivalent to $1=\sum_{i} r_{i} \operatorname{tr}(E|i\rangle\langle i|)=\sum_{i} r_{i}\langle i|E| i\rangle$. Since always $1=\sum_{i} r_{i}$ holds true, the basic certainty relation is further equivalent to $0=\sum_{i} r_{i}(1-\langle i|E| i\rangle)$. This is equivalent to $\forall i: 1=\langle i|E| i\rangle$. In turn, the last equalities are equivalent to $\forall i: 0=\left\langle i\left|E^{c}\right| i\right\rangle$, where $E^{c}$ is the ortho-complementary projector to $E$. Further equivalences give $\forall i: E^{c}|i\rangle=0$, and $\forall i: E|i\rangle=|i\rangle$, and, finally, $E Q=E \sum_{i}|i\rangle\langle i|=Q$ as claimed.

\section{B. Proof of Lemma 3.3}

Claiming the equivalence

$$
\operatorname{tr}(E \rho)=1 \Longleftrightarrow E \rho=\rho,
$$

where $E$ and $\rho$ are a projector and a density operator, respectively.

$\operatorname{tr}(E \rho)=1 \Rightarrow \operatorname{tr}\left(E^{c} \rho\right)=0$, where $E^{c} \equiv I-E, I$ being the identity operator. Further, $\operatorname{tr}\left(E^{c} \rho E^{c}\right)=0$ follows due to idempotency and commutation under the trace. The next consequence is $E^{c} \rho E^{c}=0$ because zero is the only positive operator that has zero trace. Then the Lüders lemma (cf. Remark 4.3) leads to $E^{c} \rho=0$, which, in turn, makes $E \rho=\rho$ necessary. The inverse implication is obvious.

\section{Proof of Lemma 5.2}

Claiming that if $E$ is any non-zero element of a complemented complete lattice, the set of all its lower bounds $\left\{E^{\prime}: E^{\prime} \leq E\right\}$ is also a complete lattice.

Let $\left\{E_{m}^{\prime}: m \in M\right\}$ be an arbitrary subset of $\left\{E^{\prime}: E^{\prime} \leq E\right\}$. Its glb (taken in $D(\mathscr{L})$ ) is obviously in the latter set because it is its lower bound. The lub (again taken in $D(\mathscr{\ell})$ ) is the least upper bound of the former set. Since also $E$ is an upper bound of the same set, the lub must be a lower bound of $E$ and hence it must belong to the former set.

Naturally, the claim that is symmetric to the just proved one with respect to taking the complement is proved symmetrically. It reads: Every set $\left\{E^{\prime}: E^{\prime} \geq E\right\}$ of all upper bounds of a given element $E$ of a complemented complete lattice is also a complete lattice.

Proof of the claim that the lub of a subset of a complete lattice is the same as the glb of all its upper bounds.

\section{When lub Equals glb}

Let $\left\{E_{m}: m \in M\right\}$ be a given subset, and let $\mathcal{F}$ be the set of all its upper bounds. We show that the glb $\wedge_{F \in \mathcal{F}}$ is the lub of the former set. Since each $E_{m}$ is a lower bound of $\mathcal{F}$, one has $\forall m: E_{m} \leq \wedge_{F \in \mathcal{F}}$. Thus, $\wedge_{F \in \mathcal{F}}$ is an upper bound for $\left\{E_{m}: m \in M\right\}$, hence it must belong to $\mathcal{F}$. Being the glb of $\mathcal{F}$, it is a lower bound of $\mathcal{F}$, and hence it is the least upper bound of $\left\{E_{m}: m \in M\right\}$ as claimed.

\section{References}

[1] G. Lüders, “Über die Zustandsanderung durch den Messprozess (On change of state in measurement)," Annalen der Physik (Leipzig), vol. 8, pp. 322-328, 1951.

[2] A. Messiah, Quantum Mechanics, vol. I, chapter VIII, subsection I. 2, North Holland, Amsterdam, The Netherlands, 1961. 
[3] C. Cohen-Tannoudji, B. Diu, and F. Laloë, Quantum Mechanics, John Wiley \& Sons, New York, NY, USA, 1977, Fifth Postulate.

[4] J. von Neumann, Mathematical Foundations of Quantum Mechanics, Princeton University Press, Princeton, NJ, USA, 1955.

[5] F. Herbut, "Derivation of the change of state in measurement from the concept of minimal measurement," Annals of Physics, vol. 55, no. 2, pp. 271-300, 1969.

[6] F. Herbut, "Minimal-disturbance measurement as a specification in von Neumann's quantal theory of measurement," International Journal of Theoretical Physics, vol. 11, no. 3, pp. 193-204, 1974.

[7] F. Herbut, "Derivation of the quantum probability law from minimal non-demolition measurement," Journal of Physics A, vol. 40, no. 34, pp. 10549-10555, 2007.

[8] A. Khrennikov, "The role of von Neumann and Lüders postulates in the Einstein, Podolsky, and Rosen considerations: comparing measurements with degenerate and nondegenerate spectra," Journal of Mathematical Physics, vol. 49, no. 5, Article ID 052102, 2008.

[9] A. Khrennikov, "von Neumann and Luders postulates and quantum information theory," International Journal of Quantum Information, vol. 7, no. 7, pp. 1303-1311, 2009.

[10] A. Y. Khrennikov, "Bell's inequality, Einstein, Podolsky, Rosen arguments and von Neumann's projection postulate," Laser Physics, vol. 19, no. 2, pp. 346-356, 2009.

[11] F. Herbut, "On state-dependent implication in quantum mechanics," Journal of Physics A, vol. 27, no. 22, pp. 7503-7518, 1994.

[12] F. Herbut, "More on the state-dependent implication in quantum mechanics," Journal of Physics A, vol. 29, pp. 467-469, 1996.

[13] F. Herbut, "On state-dependent implication in quantum-mechanical distant correlations," Journal of Physics A, vol. 29, no. 10, pp. 2365-2371, 1996.

[14] G. Birkhoff, Lattice Theory, American Mathematical Society, Providence, RI, USA, 1940.

[15] P. R. Halmos, A Hilbert Space Problem Book, Van Nostrand Comp, Princeton, NJ, USA, 1967. 


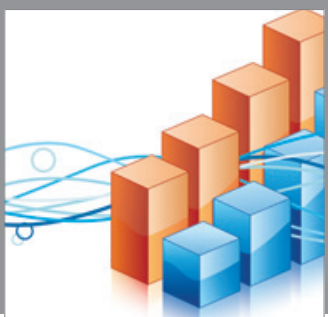

Advances in

Operations Research

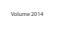

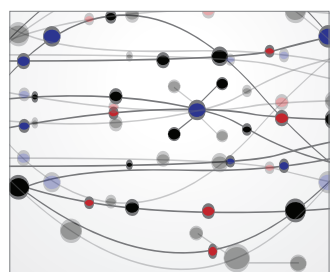

\section{The Scientific} World Journal
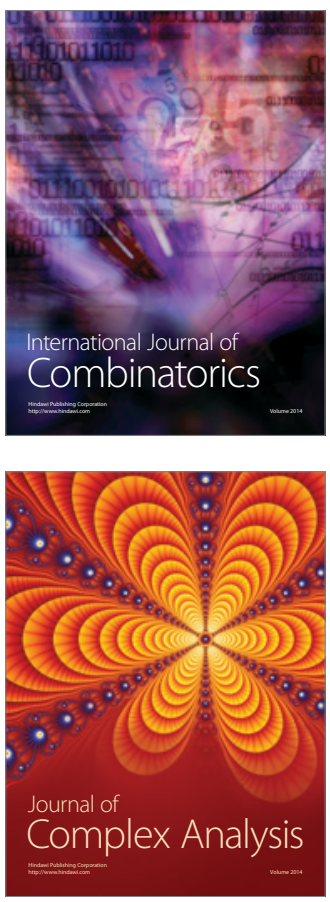

International Journal of

Mathematics and

Mathematical

Sciences
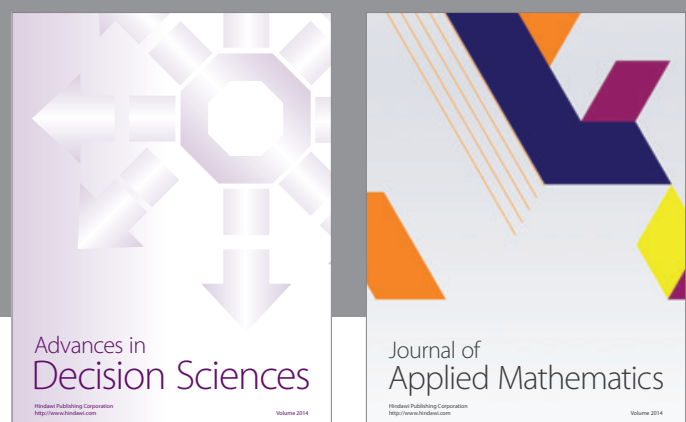

Journal of

Applied Mathematics
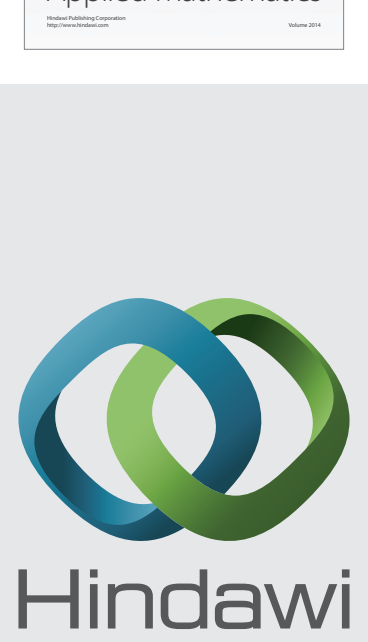

Submit your manuscripts at http://www.hindawi.com
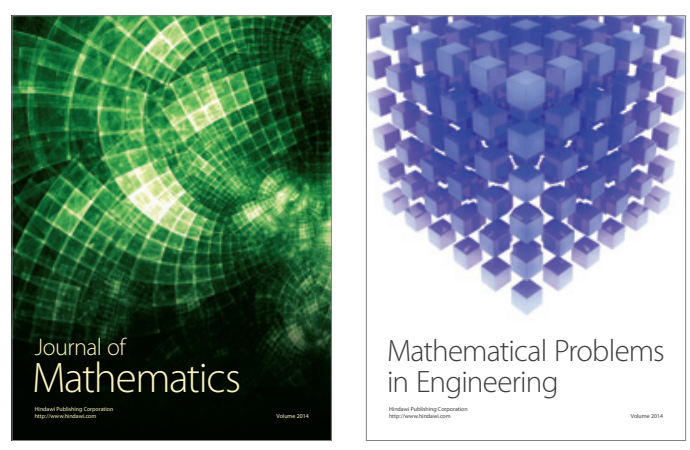

Mathematical Problems in Engineering
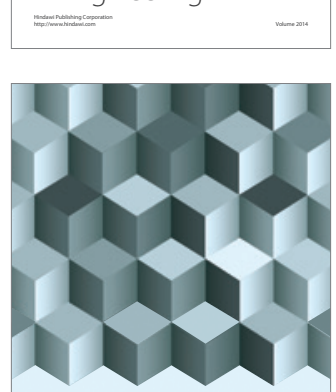

Journal of

Function Spaces
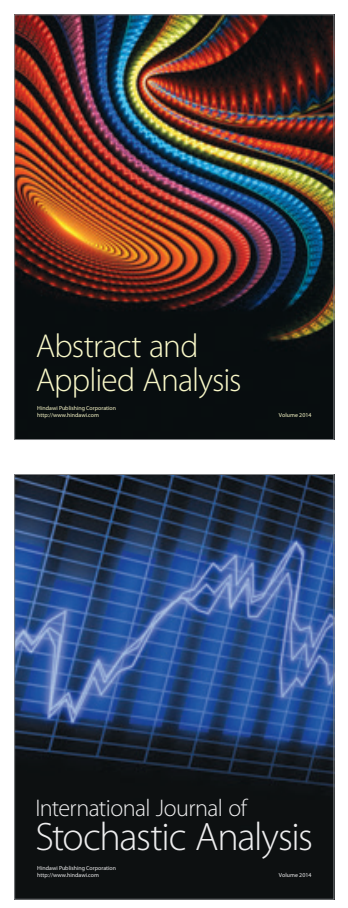

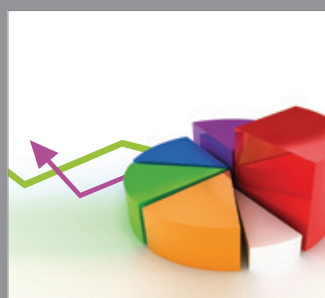

ournal of

Probability and Statistics

Promensencen
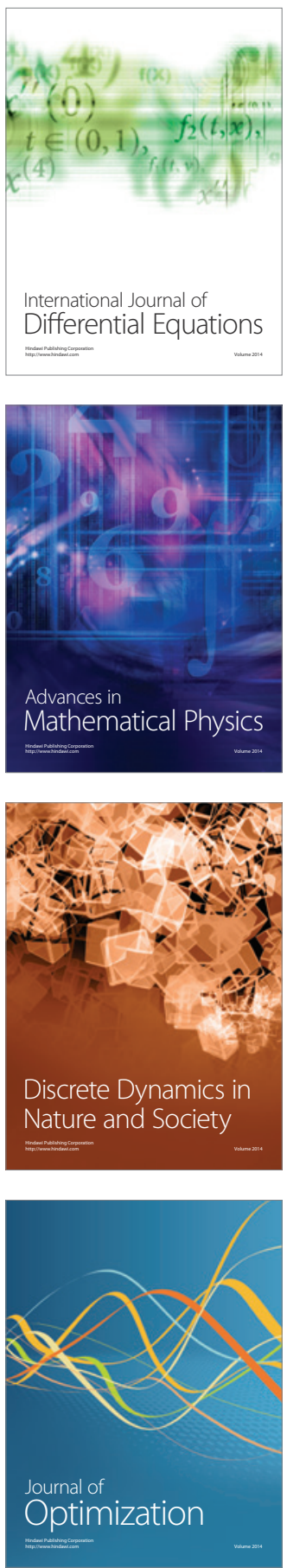